\title{
Cortical activity, kinematics and trunk muscles activity response to pelvis movements during unstable sitting
}

\author{
Ieva Aleknaite-Dambrauskiene*, Aurelijus Domeika and Arunas Zvironas \\ Institute of Mechatronics, Kaunas University of Technology, Kaunas, Lithuania
}

Received 16 August 2021

Accepted 21 September 2021

\begin{abstract}
.
BACKGROUND: Balance control is a leading component of human motor activities and its impairment is associated with an increased risk of falling, lower back pain due to impaired motor control mechanism. Prolonged sitting position at workplace is one of the risk factors of reduced postural control and lower back pain.

OBJECTIVE: To evaluate theta and alpha waves cortical activity, trunk muscles activity and kinematics in static sitting, dynamic sitting on different platforms: simple wobble board (WB) and wobble board on bearing surface (WBB).

METHODS: The kinematics of body segments, electromyography of five trunk muscles, electroencephalography of 32 scalp electrodes were measured during balance tasks in sitting position for 17 subjects with continuous seated position at workplace. RESULTS: Cortical power on WBB1 increase in fronto - central $(p<0.05)$ region while on WBB2 increase in centro - parietal region $(p<0.05)$. WBB2 increase more muscles compared with WB2. The amplitude of movement of ASIS, Th10 can be seen lower on WBB compared with WB $(p<0.05)$.

CONCLUSIONS: The study shows that WBB can increase personalized sitting and improve trunk motor control during hours of prolonged sitting.
\end{abstract}

Keywords: Electromyography, electroencephalography, occupational sitting, trunk control, wobble board

\section{Introduction}

A potential mechanism which is associated with lower back pain is known as insufficient lumbar stability [1], reduced proprioception, which causing the impaired postural control [2]. Trunk postural control is often assessed when using unstable seats to perform the sitting tasks [3]. The larger centerof-pressure while sitting on unstable seat indicates the poorer postural control [3,4]. Balance control is a leading component of human every day motor activities such as standing, walking or sitting and its impairment is associated with an increased risk of falling [5,6], functional independence [6], lower back pain due to impaired motor control mechanism. A complex interplay between the sensory and the motor systems allows a good control on posture and balance [5]. Efficient control of the center of mass

\footnotetext{
${ }^{*}$ Corresponding author: Ieva Aleknaite-Dambrauskiene, Institute of Mechatronics, Kaunas University of Technology, Studentu str. 56, Kaunas, 51424, Lithuania. Tel.: +370 37 300913; E-mail: ieva.aleknaite-dambrauskiene@ktu.lt.
} 
by proper muscle activation is essential in maintaining a good equilibrium in standing and sitting. Thus, reduced somatosensory input can affect the postural control in lower back pain.

In many studies there are talking and experimenting about increment of dynamic sitting via destabilization of support surface [6-8] and mostly this dynamic sitting or standing is explained as external perturbation [6] to reach physiological changes. Also, other dynamic sitting is reached via internal perturbation $[9,10]$ when individuals should maintain balance using external force (muscles). The important features of unstable chairs with movable seat are that it forces of the pelvis movement and increases muscle activity [6].

Moreover, studies analyzing electroencephalography (EEG) signals during external balance perturbations showed higher demands on balance control. This is associated with higher theta activity located in the medial fronto - central cortical area [7,11]. Theta activity increment during balance tasks is not only in the fronto - central cortex but also include centro - parietal regions [11] which is involved in sensory integration and sensorimotor coordination. The functional significance of frontal and parietal brain areas involvement during balance control is supported by longitudinal studies on the effects of balance training on neural structure [12]. Gray matter volume increased in frontal and parietal cortical areas after six weeks of continuous balance training and this was correlated with improvements in balance performance [12]. Also, it is important to mention, that subjects explored and evaluated different movement coordination patterns to improve performance in the dynamic balance tasks [12]. Various components of motor learning as a means to induce neuroplasticity [13]. Here we focus on evaluation of the theta and alpha waves cortical activity, trunk muscles activity and kinematics in static sitting, dynamic sitting on different platforms: simple wobble board (WB) and wobble board on bearing surface (WBB). We hypothesize that on custom - made wobble board on bearing surface pelvic motions will be more challenging according to trunk muscles activity, cortical activity, and kinematics parameters, comparing with simple wobble board.

\section{Materials and methods}

\subsection{Participants}

Seventeen subjects with non-specific lower back pain and continuous seated position at workplace were involved in the study. The subjects were $28 \pm 5.22$ years old and $177 \pm 9.4 \mathrm{~cm}$ tall, $71.5 \pm 12.81 \mathrm{~kg}$ weight.

All participants were verbally informed about the protocol and read and signed a consent form. Ethical approval (2020-06-17, No. BE-2-34) was obtained from the Regional ethics committee (Lithuanian University of Health Sciences). The Helsinki Declaration (1964) and its later amendments were also followed.

\subsection{Protocol overview}

Three different equipment were used for experiment: regular chair, wobble board (WB) and custom designed wobble board on bearing surface (WBB) (Fig. 1). The tasks were divided into 3 groups: static sitting, anterior and posterior pelvis movement on wobble board (WB1) and on wobble board on bearing surface (WBB1), lateral - medial pelvis movement on wobble board (WB2) and on wobble board on bearing surface (WBB2). There were 5 tasks and each of them was performed once and lasted for $60 \mathrm{~s}$ with the break between them. During these tasks simultaneously the kinematics of body segments, electromyography (EMG) of five trunk muscles, electroencephalography (EEG) of 32 scalp electrodes 


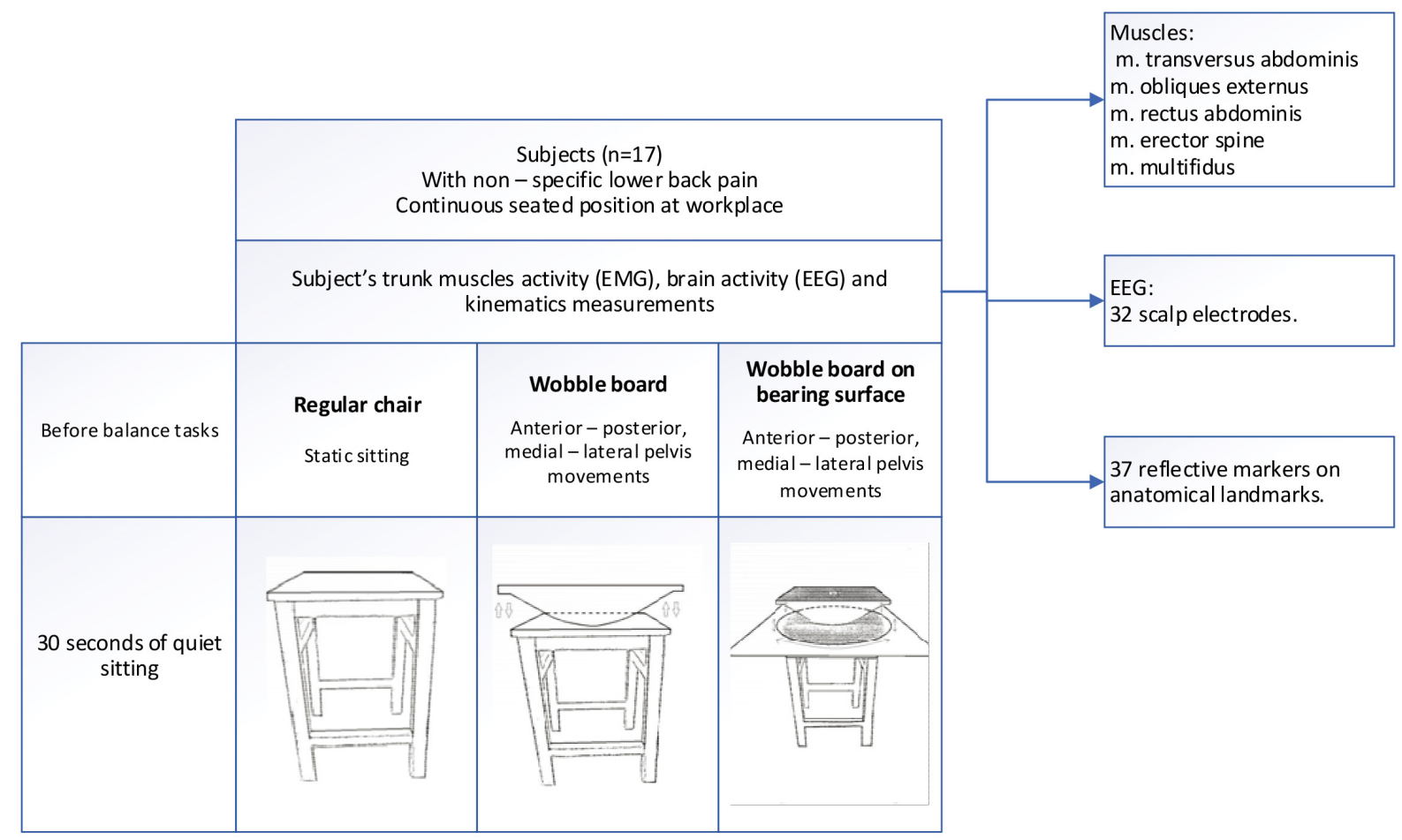

Fig. 1. Experimental protocol.

were measured. Before the balancing movements, all subjects sat quietly for $30 \mathrm{~s}$, Fig. A.1. While subjects performed the tasks in the different experimental conditions, a 3D motion capture system was used to record the trajectory of the calibrated anatomical systems technique. Reflective markers were placed on the spine, arms, pelvis, knees, ankles and feet. The locations of the reflective markers were collected using 10 high-speed infrared cameras recording at $300 \mathrm{~Hz}$ For one person five tasks were assigned and 5 trunk muscles activity, 13 EEG electrodes and 3 kinematic points were analyzed after experimental measurements. The EMG, EEG and kinematic data were recorded simultaneously.

\subsection{Data acquisition}

\subsubsection{Platform movements}

A custom - made wobble board on bearing surface was made, Fig. 2 which was showed in a previous research [14]. This unstable seat has 3 degrees of freedom (DOF), anterior - posterior, lateral - medial movements. Wobble board on bearing surface is made with 6 small bearings beneath the top surface and can move freely in all directions.

\subsubsection{Electromyography data acquisition processing and analysis}

The trunk muscles activity was measured during the balance tasks by surface electromyography (sEMG) (Noraxon MR3.6). Bipolar sEMG activity was recorded using surface electrodes from the right side of: $\mathrm{m}$. transversus abdominis (TA) $2 \mathrm{~cm}$ inferior and medial to the anterior superior iliac spine and parallel to the fibers of the TA [15], m. obliques externus (OE), m. rectus abdominis (RA) $4 \mathrm{~cm}$ to the side from the umbilicus, lower border of electrode at umbilicus level [15], m. erector spine (thoraco - lumbar part) 


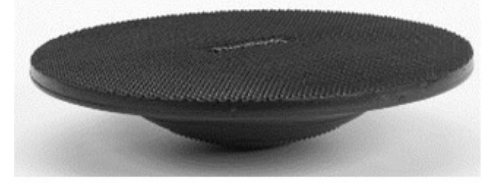

a)

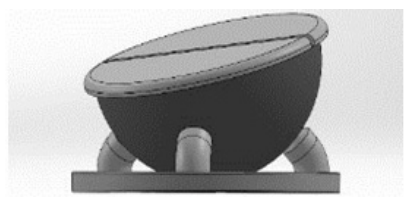

b)

Fig. 2. a) A simple wobble board and b) wobble board placed on bearing surface.

(ES) $5 \mathrm{~cm}$ lateral to T9 spinous process [16], m. multifidus (MF) aligned with a line from caudal tip posterior spina iliaca superior to the interspace between L1 and L2 interspace at the level of L5 spinous process [17]. Before the surface electrodes placement on the skin was shaved, swabbed and rubbed with alcohol to reduce skin impedance. The sampling rate was set to $1500 \mathrm{~Hz}$.

For further calculations and results comparison between participants, maximum voluntary isometric contraction (MVIC) was performed for each muscle and the sEMG amplitude recorded at the same time. MVIC was performed for every muscle three times, trial lasts 5 seconds with the rest interval between every trial. Mean MVIC EMG amplitude was calculated for each of the three, 5 seconds windows and these data were used for proportion calculations to get the \%MVIC. The data obtained from the task procedures of the pelvis were converted to percent according proportion calculation.

After measurement, the offline data processing of electromyography was done for all subjects. sEMG data were band-pass filtered (the frequency range $5-500 \mathrm{~Hz}$ ) then full wave rectified and smoothened. The amplitude of sEMG data was normalized using the mean dynamic activity method [18].

\subsubsection{Electroencephalography data acquisition processing and analysis}

Electroencephalography was recorded from 32 scalp locations overlying the whole scalp (Fp1, Fp2, F7, F3, Fz, F4, F8, FC5, FC1, FC2, FC6, AF3, AF4, T7, C3, Cz, C4, T8, CP5, CP1, CP2, CP6, P7, P3, $\mathrm{Pz}, \mathrm{P} 4, \mathrm{P} 8, \mathrm{PO} 7, \mathrm{PO} 3, \mathrm{PO} 4, \mathrm{PO}, \mathrm{Oz}$ ) [7] and equally distributed over both hemispheres according to the International 10:20-system, using a wireless EEG amplifier, g. Nautilus (g.tec medical engineering GmbH, Austria). Electrode PO8 was placed directly on mastoid bone beneath the EEG cap. The referent position was placed at the right ear. Active electrode sites were prepared by rubbing the scalp with a blunt-tipped needle, which was subsequently used to apply conductive electrode gel (g.gamma gel). Mastoid electrode site was additionally prepared by scrubbing the skin with an alcohol pad [8]. Impedances at primary electrode sites (i.e., mastoids) were $<10 \mathrm{k} \Omega$ before the start of data collection. All other active electrode sites were similarly prepared, but impedance values were not generally $<10 \mathrm{k} \Omega$ before the start of data collection. For the female subject with longer hair, the impedance at several electrode sites were maintained at $50 \mathrm{k} \Omega$ level. The sampling rate was set to $250 \mathrm{~Hz}$. Before started to measure the EEG signal, the band-pass filter was set to $0.5-100 \mathrm{~Hz}$ and the notch filter was set to $50 \mathrm{~Hz}$ [19]. The signals were measured using Simulink programmable block modules.

During measurement and after that data were collected and processed using g.BSanalyze software package for Matlab. The EEG data were first high-pass filtered (Butterworth filter, highpass: $0.5 \mathrm{~Hz}$ ) to remove noise in the lower frequencies. A semiautomatic algorithm was used to exclude segments contaminated by artifacts. For the remaining segmented data of each balance condition an Independent Component Analysis (ICA) algorithm implemented in g.BSanalyze was used for ICA decomposition [7]. Segmented data were then baseline corrected.

To investigate cortical activity changes on two different unstable surfaces, spectral power values for theta $(3-7 \mathrm{~Hz})$ and alpha $(8-13 \mathrm{~Hz})$ frequency bands were calculated (Hanning window).

According to previous studies $[7,20]$ regions of interest were defined as follows: frontal (F3, Fz, F4), fronto - central (FC1, FC2, C3, Cz, C4), centro - parietal (CP1, CP2, P3, Pz, P4). 

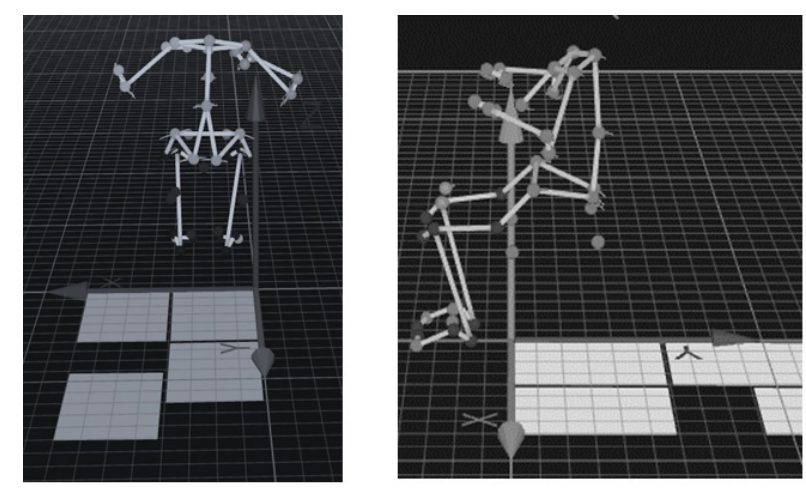

Fig. 3. Full body kinematics. Anatomical points amplitude of movement while sitting on wobble board on bearing surface (WBB) and wobble board (WB) at the same time performing lateral - medial and anterior - posterior movements.

\subsubsection{Kinematic data}

Three-dimensional kinematic data were recorded by motion capture with 10 cameras (Oqus7+ Qualisys $\mathrm{AB}$, Gothenburg, Sweden) and the Qualysis Track Manager software at a sampling frequency of $300 \mathrm{~Hz}$. The Helen-Hayes marker set was used for measuring full - body kinematics, Fig. 3. For this study two anatomical points were of major interest: pelvis point - anterior superior iliac spine (ASIS) and spine region point $10^{\text {th }}$ vertebra of thoracic spine. The amplitudes of these anatomical points were calculated during pelvis movements (lateral - medial, anterior - posterior) on two different surfaces from the direct kinematics.

\subsection{Statistical analysis}

The data of 17 participants were analysed with statistical package SPSS version 23.0. For a small sample, the nonparametric Wilcoxon test was used. The data level for statistical significance was set at $p<0.05$. The data were presented as median $x_{m e}=(\bar{x} \pm S D)$. Descriptive statistics and Spearman correlation coefficients were calculated to determine the amount of shared variance between dependent variables. When participants sat on two different unstable surfaces the anatomical point amplitude of movement and EEG signals changes were calculated to see the possible correlation between these two data sets.

\section{Results}

\subsection{Electromyography $(E M G)$}

\subsubsection{Muscles activity change on different sitting surfaces}

The increment of MF, EO and TA muscles activity were observed on WBB1 compared with WB1, Fig. B.1, however these results were not statistically significant. Except ES muscle, which activity on WBB1 was $2.45(3.35 \pm 3.74) \%$ and on WB1 $0.79(1.56 \pm 5.38) \%$. result was statistically significant $(Z=-2.430, p=0.015)$.

Significant differences of MF, ES and EO activity change on a lateral - medial pelvic movement in sitting on WB and WBB was observed, Fig. 4. Muscles activity change was measured from static sitting. The MF muscle activity on WBB2 was $8.52(10.79 \pm 7.52) \%$. the increment was statistically significant 


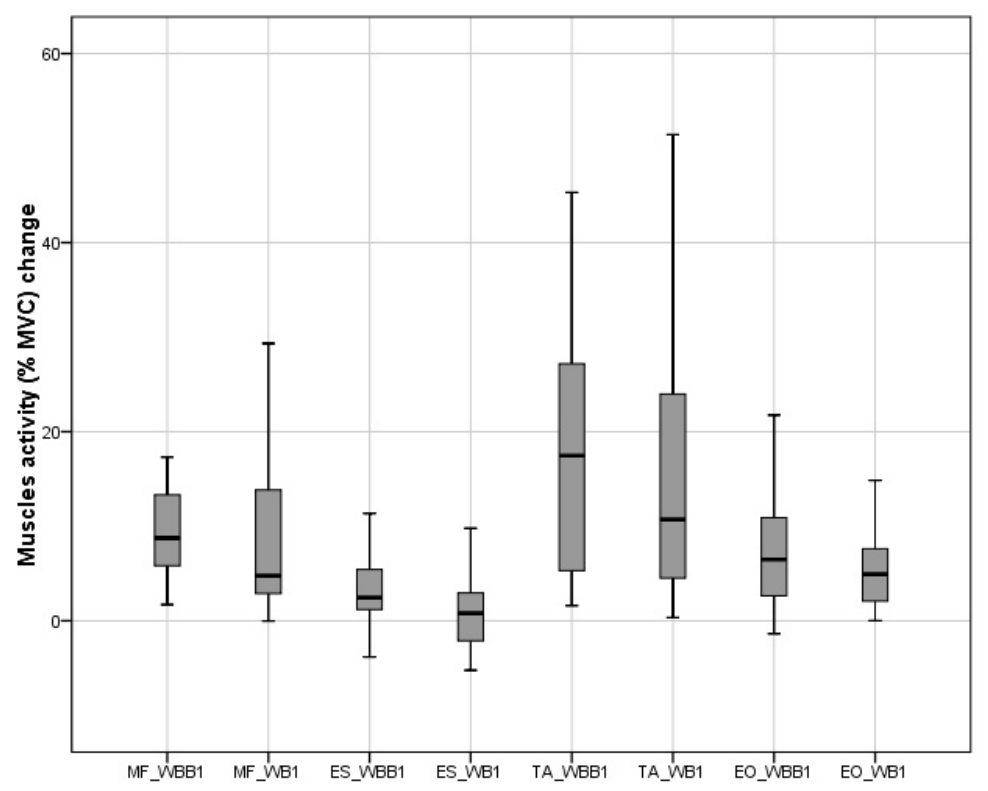

Fig. 4. Muscles activity change in anterior - posterior motion on wobble board (WB1) and muscles activity in anterior - posterior motion on wobble board on bearing surface (WBB1).

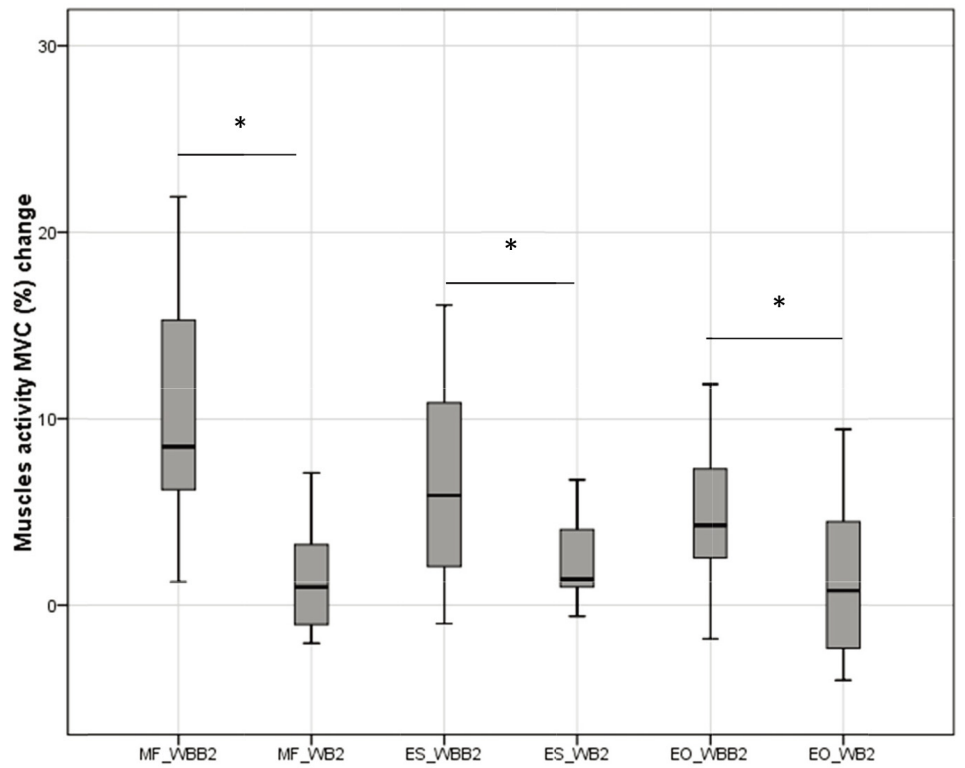

Fig. 5. Muscles activity change in lateral - medial motion on wobble board (WB2) and muscles activity in lateral - medial motion on wobble board on bearing surface (WBB2).

$(Z=-3.124, p=0.002)$ when compared with WB2 $1.24(1.95 \pm 5.75) \%$. Also, the superficial muscle ES activity on WBB2 increased by $7.98(7.60 \pm 5.33) \%$, however, on WB2 increment was smaller $1.35(2.56 \pm 3.26) \%$. The difference of ES muscles on WBB2 and WB2 was statistically significant $(Z=-3.067, p=0.002)$. Abdominal wall muscle EO activity on WBB2 was $4.87(4.81 \pm 3.73) \%$, 
Table 1

PSD of alpha wave in frontal, fronto - central, centro - parietal regions. The comparison was made next: Static sitting (SS) with anterior - posterior movement on wobble board on bearing surface (WBB1); Static sitting (SS) with anterior - posterior movement on wobble board (WB1); Static sitting (SS) with lateral - medial movement on wobble board on bearing surface (WBB2); Static sitting (SS) with lateral - medial movement on wobble board (WB2). Significant interactions are highlighted in bold type

\begin{tabular}{|c|c|c|c|c|c|c|}
\hline & \multicolumn{2}{|l|}{ Frontal region } & \multicolumn{2}{|l|}{ Fronto - central region } & \multicolumn{2}{|l|}{ Centro - parietal region } \\
\hline & Median & $\begin{array}{c}p \\
\text { value }\end{array}$ & Median & $\begin{array}{c}p \\
\text { value }\end{array}$ & Median & $\begin{array}{c}p \\
\text { value }\end{array}$ \\
\hline WBB1 & $163.85(210.07 \pm 119.71) \mu V^{2}$ & 0.007 & $200.88(220.46 \pm 186.96) \mu V^{2}$ & 0.01 & $163.93(162.27 \pm 99.15) \mu V^{2}$ & 0.041 \\
\hline WB1 & $99.08(134.67 \pm 89.56) \mu \mathrm{V}^{2}$ & 0.05 & $175.06(199.12 \pm 165.55) \mu V^{2}$ & 0.019 & $122.34(131.52 \pm 70.62) \mu \mathrm{V}^{2}$ & 0.182 \\
\hline WBB2 & $113.47(154.62 \pm 116.64) \mu V^{2}$ & 0.004 & $119.05(201.50 \pm 176.85) \mu V^{2}$ & 0.015 & $110.98(218.34 \pm 199.42) \mu V^{2}$ & 0.028 \\
\hline WB2 & $106.47(140.30 \pm 140.17) \mu \mathrm{V}^{2}$ & 0.131 & $132.34(163.34 \pm 154.56) \mu V^{2}$ & 0.034 & $115.11(150.02 \pm 113.44) \mu \mathrm{V}^{2}$ & 0.152 \\
\hline SS & $58.87(72.61 \pm 51.16) \mu \mathrm{V}^{2}$ & & $91.87(93.23 \pm 67.63) \mu \mathrm{V}^{2}$ & & $82.92(88.92 \pm 42.53) \mu \mathrm{V}^{2}$ & \\
\hline
\end{tabular}

Table 2

PSD of theta wave in frontal, fronto - central, centro - parietal regions. The comparison was made next: Static sitting (SS) with anterior - posterior movement on wobble board on bearing surface (WBB1); Static sitting (SS) with anterior - posterior movement on wobble board (WB1); Static sitting (SS) with lateral - medial movement on wobble board on bearing surface (WBB2); Static sitting (SS) with lateral - medial movement on wobble board (WB2). Significant interactions are highlighted in bold type

\begin{tabular}{|c|c|c|c|c|c|c|}
\hline & \multicolumn{2}{|l|}{ Frontal region } & \multicolumn{2}{|l|}{ Fronto - central region } & \multicolumn{2}{|l|}{ Centro - parietal region } \\
\hline & Median & $\begin{array}{c}p \\
\text { value }\end{array}$ & Median & $\begin{array}{c}p \\
\text { value }\end{array}$ & Median & $\begin{array}{c}p \\
\text { value }\end{array}$ \\
\hline WBB1 & $159.88(223.69 \pm 114.41) \mu V^{2}$ & 0.005 & $218.94(226.64 \pm 137.71) \mu V^{2}$ & 0.005 & $122.87(220.90 \pm 193.40) \mu V^{2}$ & 0.022 \\
\hline WB1 & $109.08(145.23 \pm 87.69) \mu V^{2}$ & $\mathbf{0 . 0 3 3}$ & $147.15(192.28 \pm 192.12) \mu V^{2}$ & 0.011 & $129.24(206.92 \pm \mathbf{2 0 6 . 2 4}) \mu V^{2}$ & 0.002 \\
\hline WBB2 & $110.66(221.19 \pm 254.39) \mu V^{2}$ & 0.005 & $141.91(267.26 \pm 316.74) \mu V^{2}$ & 0.005 & 247.01 $(287.61 \pm 220.59) \mu V^{2}$ & 0.022 \\
\hline WB2 & $99.16(161.58 \pm 194.60) \mu V^{2}$ & $\mathbf{0 . 0 3 3}$ & $127.66(167.49 \pm 146.84) \mu V^{2}$ & 0.011 & $132.06(172.78 \pm 150.48) \mu V^{2}$ & 0.002 \\
\hline SS & $60.73(74.30 \pm 46.73) \mu \mathrm{V}^{2}$ & & $55.81(75.86 \pm 42.32) \mu \mathrm{V}^{2}$ & & $62.94(68.32 \pm 30.69) \mu \mathrm{V}^{2}$ & \\
\hline
\end{tabular}

on WB2 was $0.77(0.44 \pm 5.44) \%$, the difference was statistically significant $(Z=-2.166, p=0.03)$ Fig. 5.

\subsection{Electroencephalography (EEG)}

\subsubsection{Alpha power spectral density (PSD)}

Alpha wave power spectral density (PSD) was calculated in two different motions: anterior - posterior and medial - lateral on different sitting surfaces: wobble board and wobble board on bearing surface. These results were compared from the static sitting and represented in Table 1.

\subsubsection{Theta power spectral density (PSD)}

Theta wave power spectral density was calculated in two different motions: anterior - posterior and medial - lateral on different sitting surfaces: wobble board and wobble board on bearing surface. These results were compared from the static sitting and represented in Table 2.

\subsection{Kinematics}

Significant differences $(Z=-3.408, p=0.001)$ were observed in the displacement of anterior posterior motion of anterior superior iliac spine (ASIS) on WBB1 $43.22(43.59 \pm 16.68)$ the amplitude of movement was smaller than on WB1 95.27 (93.53 \pm 32.52$)$. Also, the marker on the thoracic spine (Th10) 


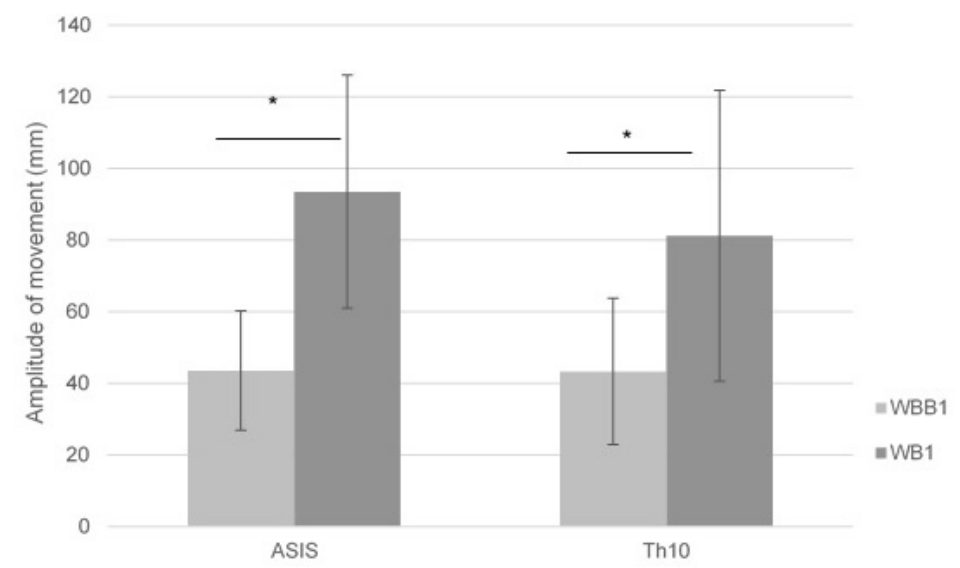

Fig. 6. Mean of ASIS and Th10 amplitude of movement in anterior - posterior pelvic motion on wobble board on bearing surface (WBB1) and wobble board (WB1).

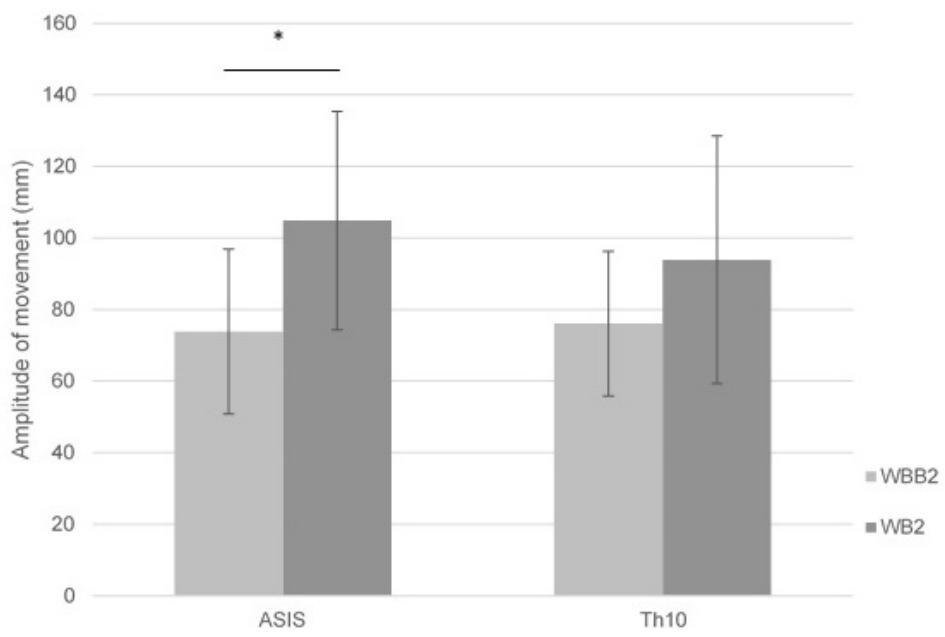

Fig. 7. Mean of ASIS and Th10 amplitude of movement in lateral - medial pelvic motion on wobble board on bearing surface (WBB2) and wobble board (WB2).

displacement was analysed in anterior - posterior motion, and the significant difference $(Z=-3.332$, $p=0.001)$ was found on WBB1 the displacement was smaller $36.96(43.32 \pm 20.48)$ compared with WB1 75.14 (81.23 \pm 40.66), Fig. 6.

Significant differences $(Z=-3.680, p<0.001)$ were observed in the amplitude of movement of lateral - medial motion of anterior superior iliac spine (ASIS) on WBB1 $69.65(73.84 \pm 23.02) \mathrm{mm}$ the amplitude of movement was smaller than on WB1 $101.24(104.85 \pm 30.54) \mathrm{mm}$. Also, the marker on the thoracic spine (Th10) amplitude of movement was analysed in lateral - medial motion, and there was no significant difference observed, Fig. 7.

\subsection{Correlation analysis}

Correlation analysis was done comparing Fz, $\mathrm{Cz}$ and Pz electrodes PSD and amplitude of movement of ASIS and Th10 anatomical points in two different movements anterior - posterior pelvic motion as well 


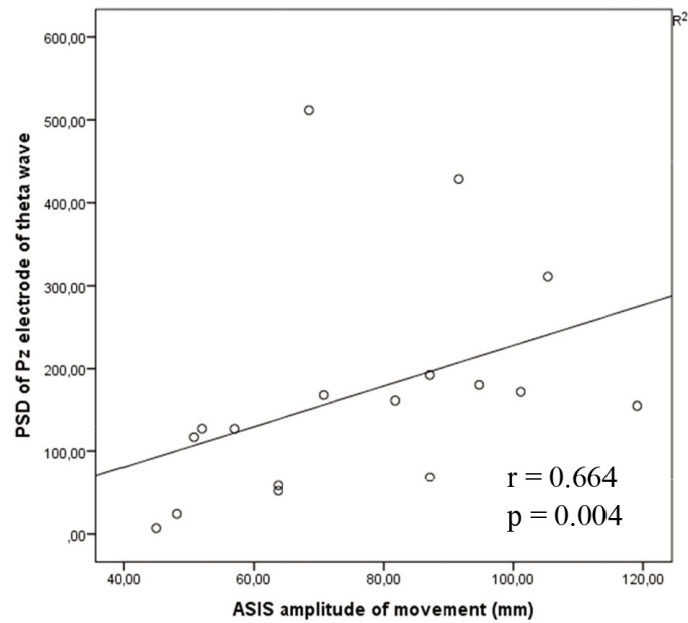

a)

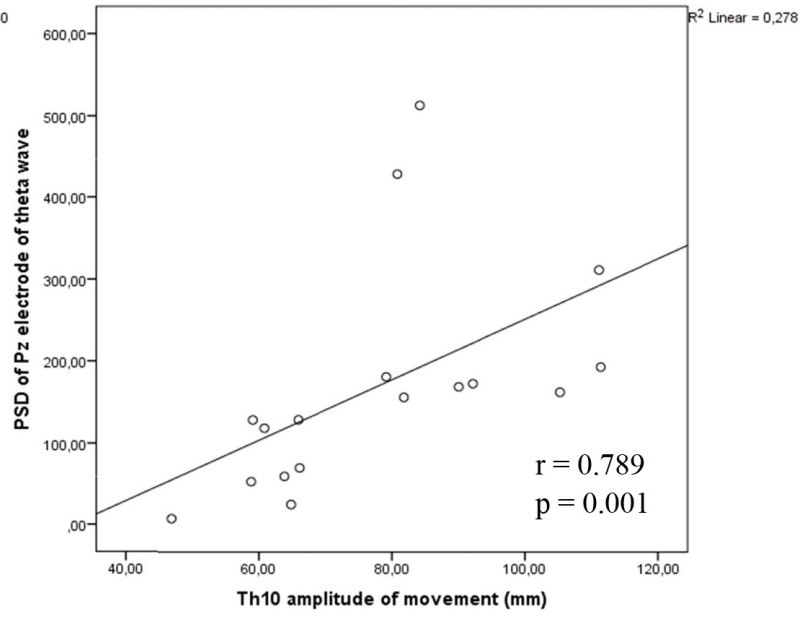

b)

Fig. 8. a) Correlations between theta power on electrode Pz and ASIS anatomical points amplitude of movement on WBB in lateral - medial movement. b) Correlations between theta power on electrode $\mathrm{Pz}$ and $\mathrm{Th} 10$ anatomical points amplitude of movement on WBB in lateral - medial movement.

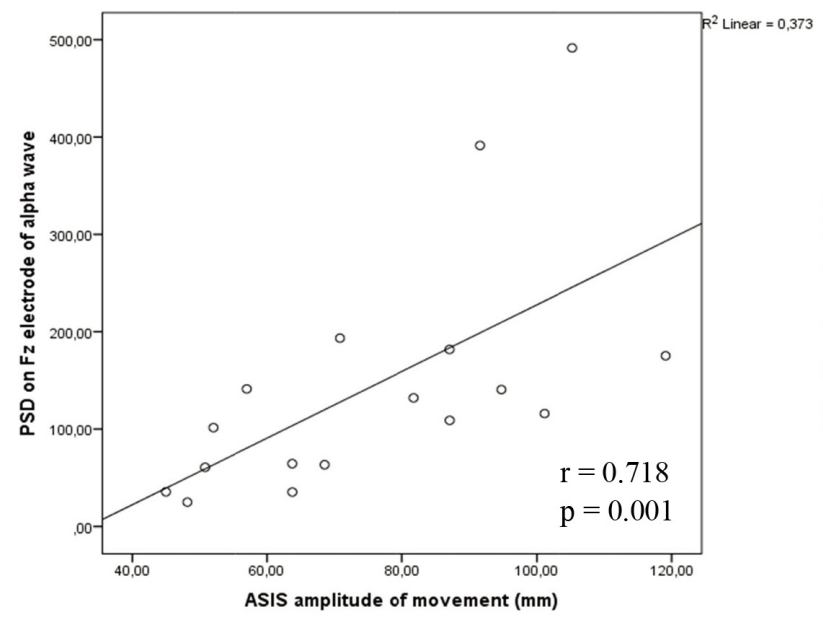

a)

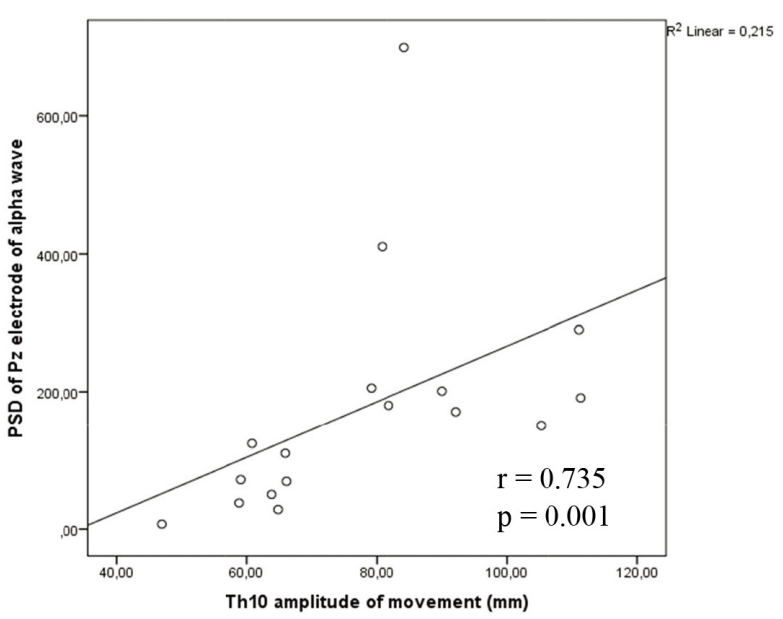

b)

Fig. 9. a) Correlations between alpha power on electrode Fz and ASIS anatomical points amplitude of movement on WBB in lateral - medial movement. b) Correlations between alpha power on electrode $\mathrm{Pz}$ and $\mathrm{Th} 10$ anatomical points amplitude of movement on WBB in lateral - medial movement.

as in lateral - medial pelvic motion on two different platforms. There were no significant correlations on wobble board. Also, we did not find any significant correlations in anterior - posterior pelvic movement.

However, the significant correlations displayed in Fig. 8a and b, were seen in lateral - medial movement on WBB of theta power on Pz electrode position with Th10 amplitude of movement $(r=0.789, p<$ $0.001)$, minor correlation was seen with ASIS amplitude of movement $(r=0.664, p=0.004)$.

Alpha power of Fz electrode correlated with Th10 amplitude of movement in lateral - medial motion on WBB $(r=0.657, p=0.02)$ as well as Cz electrode $(r=0.673, p=0.023)$ Pz electrode power $(r=$ 
0.735, $p=0.001$ ) Fig. 9a and $\mathrm{b}$. Also, correlation of alpha power in lateral - medial motion was seen in ASIS amplitude of movement and Fz electrode $(r=0.718, p=0.001)$.

\section{Discussion}

The purpose of this research was to evaluate and compare subjects' trunk muscles activity, cortical theta and alpha activity and kinematic body segments movement in static sitting and when sitting on different platforms: simple wobble board and wobble board placed on bearing surface. Scientific literature lacks research where sitting balance was analyzed as a factor that induces cortical activity. Only in a few studies position transition from sitting to standing was analyzed [21-24]. However, different sitting conditions are still little analyzed. In the present study cortical theta activity was observed on two different surfaces: wobble board and wobble board on bearing surface when performing two movements anterior posterior and lateral - medial pelvic movements. We hypothesized that on custom made wobble board on bearing surface pelvic motions will be more challenging according to trunk muscles, cortical activity and kinematic parameters, compared with simple wobble board.

The cortical theta power showed higher activity in fronto - central and centro - parietal regions. Moreover, when two different sitting surfaces were compared: static sitting and WBB, static sitting and WB, we can see that theta activity increased on both surfaces compared with static sitting $(p<0.05)$, however, it could be seen that increment was higher on custom made wobble board on bearing surface (WBB), but this was not statistically significant. From the results we can see the tendency, that custom - made wobble board on bearing surface can be more challenging than simple wobble board, but it is important to note, that this tendency was not for all participants. Because of this reason it is important to adjust balance level individually. The important correlation was found between Pz electrode position of theta power and T10 $(r=0.789)$ and ASIS $(r=0.664)$ amplitude of movement in lateral - medial pelvic movement. From the correlation analysis it may be considered that the most challenging balance for individuals mostly occurs in lateral - medial movement on wobble board on bearing surface. Studies indicated that cortical theta activity increase not only in fronto - central region [25-27] but also in centro - parietal cortex $[7,11]$. Also, other research showed theta wave increase during single leg stance and it was interpreted as involvement of the anterior cingulate cortex in monitoring postural instability [28,29]. According to studies $[20,29]$ frequency modulation in the theta, alpha, and beta bands are related with postural instability.

Another important aspect in balance tasks occurs in alpha wave excitation. When two different sitting surfaces were compared: static sitting and WBB, static sitting and WB, it could be seen that alpha activity increased on WBB compared from static sitting $(p<0.05)$ in all areas, however, when WB was compared with static sitting, the significant increment was seen only in fronto - central region $(p<0.05)$. The highest PSD (when compared: static sitting and WBB, static sitting and WB) was seen in fronto - central area compared with others and these findings coincide with other research [7,30] with the increase of task difficulty, the alpha activity also increases in frontal and central areas, from this point of view, the task on wobble board on bearing surface was more challenging. There was found important correlation of alpha wave on $\mathrm{Pz}(r=0.735)$ electrode with Th10 amplitude of movement in lateral - medial movement on wobble board on bearing surface, minor correlation was seen on $\mathrm{Cz}$ electrode position $(r=0.673)$. This correlation reveals that amplitude of anatomical point of Th10 was the optimal, to induce with balance related center activation in cortex. Studies reported the activation of the fronto-central and centro parietal cortex during self-initiated postural movement and unpredicted postural perturbations in standing 
position suggesting the supplementary motor area and the foot area of sensorimotor cortex were the possible sources in the initiating of postural movement [5,29,31].

The amplitude of movement of anatomical points ASIS and Th10 can be seen lower on wobble board on bearing surface compared with wobble board in anterior - posterior and lateral - medial movements $(p<0.05)$. The lower amplitudes of the anatomical points showed the greater physiological response and more stabilization during this movements on WBB. The experiment where balancing on wobble boards show large motion of the base of support has relatively small motion of the upper body [32]. According to amplitude of movement of anatomical points the muscles activity is higher where amplitude of movement of anatomical points are lesser. It could be because of higher efforts which were needed to do pelvic movements. Study [1] indicates the motor control challenge for the individuals is to use their lumbar spine to minimize the seat displacement, when sitting on unstable surface, with smaller movements representing better performance.

The activity increment in MF, ES and EO muscles were observed in lateral - medial movement on wobble board placed on bearing surface compared with wobble board $(p<0.05)$. According to study [33] motion in anterior - posterior directions shows stabilizing moment, but in medial - lateral directions authors did not see stiffness control subsequently identifying the cause of muscle(s) that could exhibit such behavior were not studied; and/or the CNS does not employ stiffness control in the medial - lateral directions. During anterior - posterior movement significantly increase only in ES muscle on wobble board placed on bearing surface. This custom-made wobble board on bearing surface can be used as training device which can be included in workplace. As can be seen from the other studies [34,35] where dynamic chairs are used instead of basic office chairs can be seen that amount of spinal motion in sitting increases. Moreover, trunk muscles activation does not change or increases [35-37]. So, this wobble board on bearing surface which can move in anterior - posterior and lateral - medial directions can arouse trunk muscles activity especially in lateral - medial movement.

This study is not without limitations. For instance, the sample size could be bigger, also it is important to evaluate eyes-closed condition in EEG measurements, like other studies did. Further research is needed to overcome these limitations.

\section{Conclusions}

The results of the present study indicate a global increase in theta and alpha waves PSD when performing anterior - posterior and medial - lateral movements on wobble board and on wobble board on bearing surface, which means that the balance maintenance in sitting position on WB and WBB was challenging. However, theta and alpha waves power in medial - lateral movement on wobble board on bearing surface was found to be strongly correlated to anatomical points amplitude of movement, probably reflecting higher demands on task which evokes balance perturbation. Furthermore, lateral - medial movement on wobble board on bearing surface increase more muscles activity compared with anterior - posterior movement. As previously said, the anterior - posterior movement is more stabilizing and participants with non - specific lower back pain have impaired trunk motor control and stabilization, which less is needed in lateral - medial movement. This study provides new insights of how efficiently improve trunk motor control during hours of prolonged sitting, when neuromuscular control of pelvis and lumbar spine are affected and people with non-specific lower back pain cannot move pelvis isolated, from the findings we can see that WBB can help to feel and increase the pelvis movements. Wobble board on bearing surface could be adjusted to increase trunk muscles activity, especially for those people who have impaired neuromuscular control in lumbo-pelvic region. However, more studies need to be performed, 
with bigger sample size and including eyes-open, eyes-closed condition, more accurately evaluate cortical response.

\section{Acknowledgments}

The authors would like to thank neurologist Vaidotas Gudžiūnas and physiotherapist Simona Puskunigytè-Krasuckienè.

\section{Conflict of interest}

None to report.

\section{Funding}

The authors received no financial support for the research, authorship, and/or publication of this article.

\section{References}

[1] Andreopoulou G, Maaswinkel E, Cofré Lizama LE, van Dieën JH. Effects of support surface stability on feedback control of trunk posture. Exp Brain Res. 2015; 233(4): 1079-87.

[2] AU - Williams AD, AU - Vette AH. A Vibrotactile Feedback Device for Seated Balance Assessment and Training. JoVE [Internet]. 2019; (143): e58611. Available from: https://www.jove.com/video/58611.

[3] Acasio JC, Nussbaum MA, Hendershot BD. Trunk-pelvic coordination during unstable sitting with varying task demand: A methodological study. J Biomech [Internet]. 2021; 118: 110299. Available from: doi: 10.1016/j.jbiomech.2021.110299.

[4] Acasio JC, Butowicz CM, Golyski PR, Nussbaum MA, Hendershot BD. Associations between trunk postural control in walking and unstable sitting at various levels of task demand. J Biomech [Internet]. 2018; 75: 181-5. Available from: doi: 10.1016/j.jbiomech.2018.05.006.

[5] Mierau A, Pester B, Hülsdünker T, Schiecke K, Strüder HK, Witte H. Cortical correlates of human balance control. Brain Topogr. 2017; 30(4): 434-46.

[6] Williams AD, Kumawat A, Agarwal K, Boser QA, Rouhani H, Vette AH. An instrumented wobble board for assessing and training dynamic sitting balance. 2017 IEEE Int Conf Syst Man, Cybern SMC 2017. 2017; 2017-Janua: $2249-54$.

[7] Hülsdünker T, Mierau A, Strüder HK. Higher Balance Task Demands are Associated with an Increase in Individual Alpha Peak Frequency. 2016; 9(January): 1-12.

[8] Payne AM, Hajcak G, Ting LH. Dissociation of muscle and cortical response scaling to balance perturbation acceleration. J Neurophysiol. 2019; 121(3): 867-80.

[9] Slobounov S, Hallett M, Stanhope S, Shibasaki H. Role of cerebral cortex in human postural control: An EEG study. Clin Neurophysiol. 2005; 116(2): 315-23.

[10] Larivière C, Preuss R, Ludvig D, Henry SM. Is postural control during unstable sitting a proxy measure for determinants associated with lumbar stability? J Biomech. 2020; 102: 1-9.

[11] Hülsdünker T, Mierau A, Neeb C, Kleinöder H, Strüder HK. Cortical processes associated with continuous balance control as revealed by EEG spectral power. Neurosci Lett [Internet]. 2015; 592: 1-5. Available from: http://www.scienc edirect.com/science/article/pii/S0304394015001639.

[12] Taubert M, Draganski B, Anwander A, Müller K, Horstmann A, Villringer A, et al. Dynamic properties of human brain structure: Learning-related changes in cortical areas and associated fiber connections. J Neurosci. 2010; 30(35): 11670-7.

[13] Diekfuss JA, Grooms DR, Hogg JA, Singh H, Slutsky-Ganesh AB, Bonnette S, et al. Targeted Application of Motor Learning Theory to Leverage Youth Neuroplasticity for Enhanced Injury-Resistance and Exercise Performance: OPTIMAL PREP. J Sci Sport Exerc [Internet]. 2021; (0123456789). Available from: doi: 10.1007/s42978-020-00085-y.

[14] Aleknaite-dambrauskiene I, Domeika A, Gudžiūnas V, Zaveckas V. Pilot Study of Wobble Seat on Trunk Muscles Activity and Kinematics. 2021; 27(2): 155-8. 
[15] Flowers DW, Brewer W, Ellison J, Mitchell K, Frilot C. Transversus abdominis activation does not alter gait impairments in patients with and without knee osteoarthritis. Clin Biomech [Internet]. 2021; 82(December 2020): 105270. Available from: doi: 10.1016/j.clinbiomech.2020.105270.

[16] McCook DT, Vicenzino B, Hodges PW. Activity of deep abdominal muscles increases during submaximal flexion and extension efforts but antagonist co-contraction remains unchanged. J Electromyogr Kinesiol [Internet]. 2009; 19(5): 754-62. Available from: doi: 10.1016/j.jelekin.2007.11.002.

[17] Recommendations for sensor locations in trunk or (lower) back muscles [Internet]. Available from: http://wwwseniam.org/.

[18] Freddolini M, Strike S, Lee RYW. The role of trunk muscles in sitting balance control in people with low back pain. J Electromyogr Kinesiol [Internet]. 2014; 24(6): 947-53. Available from: doi: 10.1016/j.jelekin.2014.09.009.

[19] Attia M, Hettiarachchi I, Hossny M, Nahavandi S. A time domain classification of steady-state visual evoked potentials using deep recurrent-convolutional neural networks. Proc - Int Symp Biomed Imaging. 2018; 2018-April(Isbi): 766-9.

[20] Slobounov S, Hallett M, Cao C, Newell K. Modulation of cortical activity as a result of voluntary postural sway direction: An EEG study. Neurosci Lett [Internet]. 2008/07/12. 2008 Sep 19; 442(3): 309-13. Available from: https://pubmed.ncbi. nlm.nih.gov/18639613.

[21] De Sanctis P, Butler JS, Malcolm BR, Foxe JJ. Recalibration of inhibitory control systems during walking-related dual-task interference: A Mobile Brain-Body Imaging (MOBI) Study. Neuroimage. 2014; 94: 55-64.

[22] Malcolm BR, Foxe JJ, Butler JS, De P, Tishman DR, Neurophysiology C, et al. HHS Public Access. 2016; $230-42$.

[23] Mochizuki G, Sibley KM, Cheung HJ, Camilleri JM, McIlroy WE. Generalizability of perturbation-evoked cortical potentials: Independence from sensory, motor and overall postural state. Neurosci Lett. 2009; 451(1): 40-4.

[24] Bulea TC, Prasad S, Kilicarslan A, Contreras-Vidal JL. Sitting and standing intention can be decoded from scalp EEG recorded prior to movement execution. Front Neurosci. 2014; 8(Nov): 1-19.

[25] Sipp AR, Gwin JT, Makeig S, Ferris DP. Loss of balance during balance beam walking elicits a multifocal theta band electrocortical response. J Neurophysiol. 2013; 110(9): 2050-60.

[26] Varghese JP, Beyer KB, Williams L, Miyasike-daSilva V, McIlroy WE. Standing still: Is there a role for the cortex? Neurosci Lett [Internet]. 2015; 590: 18-23. Available from: doi: 10.1016/j.neulet.2015.01.055.

[27] Slobounov SM, Teel E, Newell KM. Modulation of cortical activity in response to visually induced postural perturbation: Combined VR and EEG study. Neurosci Lett [Internet]. 2013; 547: 6-9. Available from: doi: 10.1016/j.neulet.2013.05.001.

[28] Wittenberg E, Thompson J, Nam CS, Franz JR. Neuroimaging of human balance control: A systematic review. Front Hum Neurosci. 2017; 11(April): 1-25.

[29] Varghese JP, Marlin A, Beyer KB, Staines WR, Mochizuki G, McIlroy WE. Frequency characteristics of cortical activity associated with perturbations to upright stability. Neurosci Lett [Internet]. 2014; 578: 33-8. Available from: doi: 10.1016/j.neulet.2014.06.017.

[30] Katahira K, Yamazaki Y, Yamaoka C, Ozaki H, Nakagawa S, Nagata N. EEG correlates of the flow state: A combination of increased frontal theta and moderate frontocentral alpha rhythm in the mental arithmetic task. Front Psychol. 2018; 9(MAR): 1-11.

[31] Tse YYF, Petrofsky JS, Berk L, Daher N, Lohman E, Laymon MS, et al. Postural sway and rhythmic electroencephalography analysis of cortical activation during eight balance training tasks. Med Sci Monit [Internet]. 2013 Mar 8; 19: 175-86. Available from: https://www.ncbi.nlm.nih.gov/pubmed/23470794.

[32] Roberts BWR, Vette AH. A kinematics recommendation for trunk stability and control assessments during unstable sitting. Med Eng Phys. 2019; 73: 73-6.

[33] Roberts BWR, Gholibeigian F, Lewicke J, Vette AH. Spatial and temporal relation of kinematics and muscle activity during unstable sitting. J Electromyogr Kinesiol [Internet]. 2020; 52(November 2019): 102418. Available from: doi: 10.1016/j.jelekin.2020.102418.

[34] O'Sullivan K, McCarthy R, White A, O'Sullivan L, Dankaerts W. Lumbar posture and trunk muscle activation during a typing task when sitting on a novel dynamic ergonomic chair. Ergonomics. 2012; 55(12): 1586-95.

[35] Kingma I, van Dieën JH. Static and dynamic postural loadings during computer work in females: Sitting on an office chair versus sitting on an exercise ball. Appl Ergon. 2009 Mar; 40(2): 199-205.

[36] Gregory DE, Dunk NM, Callaghan JP. Stability ball versus office chair: Comparison of muscle activation and lumbar spine posture during prolonged sitting. Hum Factors [Internet]. 2006 Mar 1; 48(1): 142-53. Available from: doi: $10.1518 / 001872006776412243$.

[37] O’Sullivan K, O'Sullivan P, O'Keeffe M, O'Sullivan L, Dankaerts W. The effect of dynamic sitting on trunk muscle activation: A systematic review. Appl Ergon [Internet]. 2013; 44(4): 628-35. Available from: doi: 10.1016/j.apergo.2012.12.006. 